\title{
Practical Surgical Techniques for Lymphadenectomy Along the Right Recurrent Laryngeal Nerve During Thoracoscopic Esophagectomy in the Prone Position
}

\author{
Taro Oshikiri, MD, Tetsu Nakamura, MD, Yukiko Miura, MD, Hiroshi Hasegawa, MD, Masashi Yamamoto, MD, \\ Shingo Kanaji, MD, Kimihiro Yamashita, MD, Yoshiko Matsuda, MD, Takeru Matsuda, MD, Yasuo Sumi, MD, \\ Satoshi Suzuki, MD, and Yoshihiro Kakeji, MD
}

Division of Gastrointestinal Surgery, Department of Surgery, Graduate School of Medicine, Kobe University, Kobe, Hyogo, Japan

\begin{abstract}
Background. In esophageal squamous cell cancer (SCC), lymphadenectomy along the right recurrent laryngeal nerve (RLN) is important for disease control. The metastatic rate was $33 \%$ and the 5-year overall survival rate of these patients was $33.3 \%,{ }^{1}$ but the risk of RLN palsy increases. ${ }^{2}$ We reported a reliable new method ('Pincers Maneuver') ${ }^{3}$ for lymphadenectomy along the right RLN during thoracoscopic esophagectomy in the prone position (TEP), and hereby present our video, aimed at providing a complete and safe dissection.

Method. The 'Pincers Maneuver' is performed for all resectable clinical stage IA-III lower, middle, or upper thoracic esophageal SCCs. Patients above clinical stage IB were treated with neoadjuvant chemotherapy. The concept of this procedure is to first exfoliate the two-dimensional membrane (lateral pedicle), which includes the right RLN, lymph nodes, and the primary esophageal artery, from the right side of the trachea toward the neck. Improved mobility of the lateral pedicle, gained by closing in from its inner and outer sides, enables easy lymphadenectomy along the right RLN toward the right inferior thyroid artery.
\end{abstract}

Electronic Supplementary Material The online version of this article (doi:10.1245/s10434-017-5879-2) contains supplementary material, which is available to authorized users.

(C) Society of Surgical Oncology 2017

First Received: 6 March 2017;

Published Online: 16 May 2017

T. Oshikiri, MD

e-mail: oshikiri@med.kobe-u.ac.jp
Results. Using this method, we performed 31 TEPs in 2016 at Kobe University Hospital. Median body mass index was $23 \mathrm{~kg} / \mathrm{m}^{2}$ (range 18-31). No right RLN palsy greater than Clavien-Dindo classification grade I was observed. On average, $5.2 \pm 2.7$ nodes were harvested along the right RLN, with a $23 \%$ metastatic rate.

Conclusions. Our method for lymphadenectomy along the right RLN during TEP is safe and practical. It provides sufficient lymph node dissection, and no right RLN palsy has been observed.

DISCLOSURE Taro Oshikiri, Tetsu Nakamura, Yukiko Miura, Hiroshi Hasegawa, Masashi Yamamoto, Shingo Kanaji, Kimihiro Yamashita, Yoshiko Matsuda, Takeru Matsuda, Yasuo Sumi, Satoshi Suzuki, and Yoshihiro Kakeji have no conflicts of interest or financial ties to disclose. This study was approved by the Institutional Review Board and the Ethics Committee of Kobe University.

\section{REFERENCES}

1. Taniyama Y, Nakamura T, Mitamura A, Teshima J, Katsura K, Abe $\mathrm{S}$, et al. A strategy for supraclavicular lymph node dissection using recurrent laryngeal nerve lymph node status in thoracic esophageal squamous cell carcinoma. Ann Thorac Surg. 2013;95:1930-7.

2. Sato Y, Kosugi SI, Aizawa N, Ishikawa T, Kano Y, Ichikawa H, et al. Risk factors and clinical outcomes of recurrent laryngeal nerve paralysis after esophagectomy for thoracic esophageal carcinoma. World J Surg. 2015;40:129-36.

3. Oshikiri T, Nakamura T, Miura Y, Yamamoto M, Kanaji S, Yamashita K, et al. A new method (the "Pincers maneuver") for lymphadenectomy along the right recurrent laryngeal nerve during thoracoscopic esophagectomy in the prone position for esophageal cancer. Surg Endosc. 2017;31:1496-504. 